\title{
A MARKOV MODEL FOR LOSS RESERVING
}

\author{
By Ole Hesselager \\ University of Copenhagen, Denmark
}

\begin{abstract}
The claims generating process for a non-life insurance portfolio is modelled as a marked Poisson process, where the mark associated with an incurred claim describes the development of that claim until final settlement. An unsettled claim is at any point in time assigned to a state in some state-space, and the transitions between different states are assumed to be governed by a Markovian law. All claims payments are assumed to occur at the time of transition between states. We develop separate expressions for the IBNR and RBNS reserves, and the corresponding prediction errors.
\end{abstract}

\section{KEYWORDS}

IBNR and RBNS reserves; marked point process; Markov chain; martingale.

\section{INTRODUCTION}

HACHEMEISTER (1980) suggested to represent the information about an unsettled claim by modelling the development as the realization of a (discrete-time) Markov chain. The predicted future claims cost for a particular claim then depends on the current state of the claim, and the state space represents the possible types of information which the company may have (or want to consider) during the development process. In this paper we adopt the ideas of HACHEMEISTER (1980) and describe the development of a claim from occurrence until final settlement as the realization of a time-inhomogeneous, continuous-time Markov chain. We extend the description by also modelling the claim occurrences - by a time-inhomogeneous Poisson process. This makes it possible to establish separate reserves for the pure IBNR (Incurred But $\underline{N}$ ot $\underline{R}$ eported) claims and the RBNS (ㄹeported B $u$ t $\underline{N}$ ot Settled) claims.

The reserving (or prediction) problem is conveniently formulated within the framework of marked (Poisson) point processes, which was advocated in the context of claims reserving by ARJAS (1989), and further developed by NORBERG (1993). In this context it is then assumed that the marks consist of the realization of a Markov chain together with the claims payments, which are assumed to occur at the times of transition between different states.

The present paper gives a time-continuous version of HACHEMEISTER's (1980) model. Our way of modelling the claims payments, however, differs from that of 
HACHEIMEISTER (1980), and hence also our formulas for the IBNR and RBNS reserves. In particular, the formulas given for the prediction errors, which are used to assess the quality of the IBNR and RBNS reserves, appear to be new.

\section{THE MODEL}

Consider a portfolio which has been observed during some time interval $[0, \tau]$, where $\tau$ represents the present moment. We denote by $K(t)$ the number of claims incurred during $[0, t]$, and by $0<T_{1}<T_{2}<\ldots$ the corresponding times of occurrence. With the $i$ th claim we associate a mark $Z_{i}$, which describes the development of that claim until final settlement. The marks are constructed as

$$
Z_{i}=Z^{\left(T_{i}\right)}
$$

where $\left\{Z^{(t)}\right\}_{t \geq 0}$ is a family of random elements. For the claims generating process we assume that

(a) $\{K(t)\}_{t \geq 0}$ is a Poisson process with intensity $\{\mu(t)\}_{t \geq 0}$, and $\left\{Z^{(t)}\right\}_{t \geq 0}$ are mutually independent and independent of $\{K(t)\}_{t \geq 0)}$.

A claim is at any point in time after occurrence assigned to one of at most countable many states, $\mathcal{S}$. Different states in the set $\mathcal{S}$ represent different types of information about the claim which the company may have. During the development process a claim may change state as new information becomes available, and (partial) payments may be made at the times of transition between states. We want the mark $Z_{i}$ to carry the information about how the $i$ th claim is classified in the course of time, and also payments being made on that claim. Thus, we let

$$
Z^{(t)}=\left\{\left\{S^{(t)}(u)\right\}_{u \geq 0},\left\{Y_{m n, j}^{(t)}\right\}_{j=1,2, \ldots,} m \neq n, m, n \in \mathcal{S}\right\},
$$

where $S^{(t)}(u) \in S$ denotes the state at time $t+u$ of a claim incurred at time $t$, and $Y_{m n, j}^{(t)}$ denotes the payment made upon the $j$ th transition from $m$ to $n$.

For a claim incurred at time $t$, transitions from $m$ to $n$ occur at time epochs $t+U_{m n, 1}^{(t)}, t+U_{m n, 2}^{(t)}, \ldots$, say. The payments $Y_{m n, j}^{(t)}$ are regarded as marks corresponding to the point process $0<U_{m n, 1}^{(t)}<U_{m n, 2}^{(t)}<\ldots$, and are constructed as

$$
Y_{m n, j}^{(t)}=Y_{m n}^{(t)}\left(U_{m n, j}^{(t)}\right)
$$

where $\left\{Y_{m n}^{(t)}(u)\right\}_{u \geq 0}$ is a family of random elements. For the development process we assume that

(b) $\left\{S^{(t)}(u)\right\}_{u \geq 0}$ is a time-inhomogeneous Markov chain with transition probabilities

$$
p_{m n}(u, v)=\mathrm{P}\left(S^{(t)}(v)=n \mid S^{(t)}(u)=m\right),
$$

and intensities

$$
\lambda_{m n}(u)=\lim _{h \rightarrow 0+} p_{m n}(u, u+h) / h
$$


The amounts $\left\{Y_{m n}^{(t)}(u)\right\}_{u \geq 0}$ are mutually independent for all $m \neq n \in S$ and $u \geq 0$, and are independent of $\left\{S^{(t)}(u)\right\}_{u \geq 0}$ with cumulative distribution function

$$
F_{m n}(y \mid u)=\mathrm{P}\left(Y_{m n}^{(t)}(u) \leq y\right)
$$

Remark 2.1. According to assumption (b), the distribution of the mark $Z^{(t)}$ corresponding to a claim incurred at time $t$ does not depend on time $t$. This assumption could be dropped without any consequences for the following - except that the intensities $\lambda_{m n}(u)$ and the distributions $F_{m n}(y \mid u)$ would then carry topscript $t$. A particular dependence on time $t$ is that where $\lambda_{m n}^{(t)}(u)=\lambda_{m n}(t+u)$ depends on calendar time $t+u$ rather than waiting time $u$ since occurrence of the claim. This may be a reasonable specification e.g. for transitions corresponding to the settlement of RBNS claims (see Examples 2 and 3 below). It is a different matter that the statistical estimation becomes more difficult in such cases. In fact, since the claims reserving problem is concerned with payments made at time epochs $t+u>\tau$, one will at time $\tau$ only be able to estimate the relevant intensities $\lambda_{m n}(t+u)$ if some parametric assumption is being made.

Example 1. In the simplest possible model,

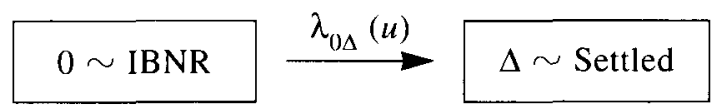

it is assumed that a claim is settled at the time of notification. Let $W$ be the waiting time until notification, and let $G(u)=\mathrm{P}(W \leq u)$. With only two states, $S=\{0, \Delta\}$, where $0 \sim$ IBNR and $\Delta \sim$ Settled, this model is trivially Markov, and the rate of settlement is $\lambda_{04}(u)=G^{\prime}(u) /(1-G(u))$.

Example 2. Consider the model,

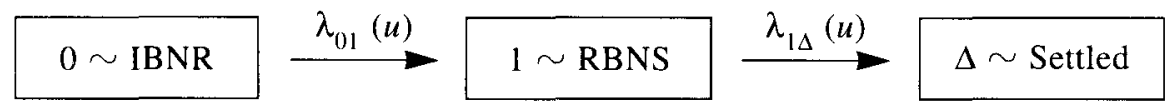

where the reporting as well as the settlement of claims is subject to a delay. The assumption (b), that the intensities $\lambda_{m n}$ depend on $u$, the time elapsed since occurrence, may seem inadequate as far as $\lambda_{1 \Delta}$ is concerned. The management might want to assume that the rate of settlement for RBNS claims is determined by the amount of resources which are allocated to claims handling department, and that $\lambda_{14}$ should therefore depend on calendar time $t+u$. As pointed out in Remark 2.1 above, this is also possible within the current framework. One could take

$$
\begin{aligned}
& \lambda_{01}^{(t)}(u)=\lambda_{01}(u), \\
& \lambda_{0 \Delta}^{(t)}(u)=\lambda_{0 \Delta}(t+u),
\end{aligned}
$$


in which case the rate of reporting depends on waiting time $u$ since occurrence and the rate of settlement depends on calendar time $t+u$.

Example 3. Consider the following example, inspired by HACHEMEISTER (1980). In some lines of business, with the possibility of having very large claims, it is customary that the claims handling department at the time of notification reviews the details concerning a claim and makes an estimate whether the ultimate claim amount is likely to exceed some prescribed limit, say DDK 200.000. If so, a case reserve (RBNS) is calculated for this claim. The company may later receive new information which causes it to revise the initial estimate. A claim which at the time of notification was judged to exceed the prescribed limit may then be re-classified as a "small" claim, and vice versa. Obviously the model could be refined by introducing more states, representing different intervals for the individual estimate (case reserve) for a claim.

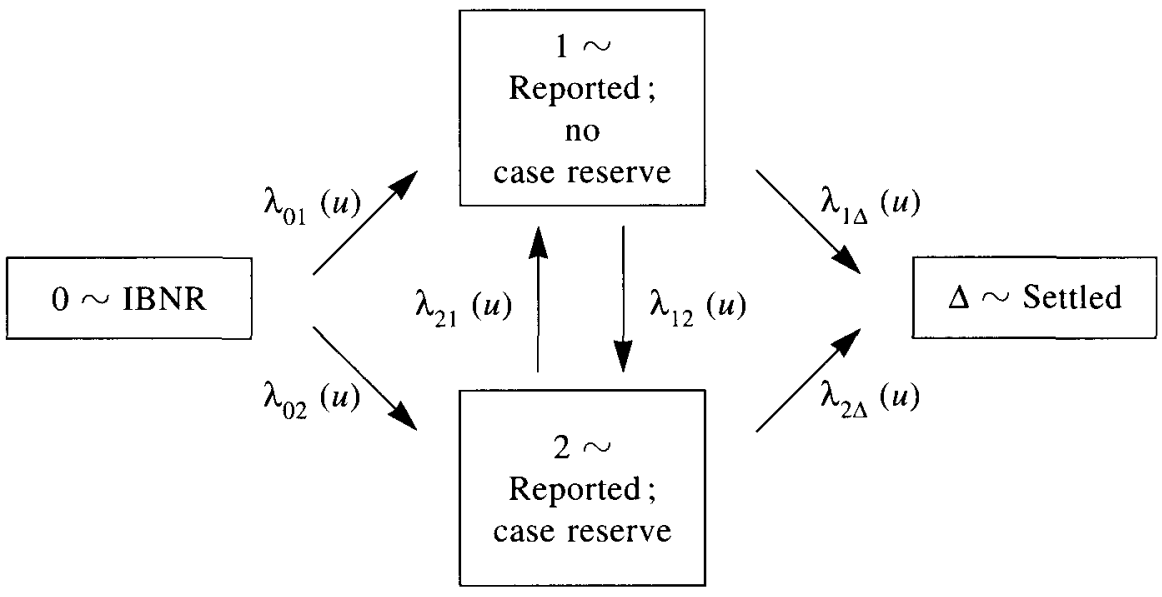

For a claim incurred at time $t$ we shall need the following quantities,

$I_{m}^{(t)}(u)=I\left(S^{(t)}(u)=m\right)$, the indicator of the event that the claim occupies state $m$ at time $t+u$,

$N_{m n}^{(t)}(u)$, the number of direct transitions from $m$ to $n$ during $[t, t+u]$, $\mathcal{H}_{u}^{(t)}=\sigma\left(\left\{S^{(t)}(v)\right\}_{0 \leq v \leq u},\left\{Y_{m n, j}^{(t)}, j=1, \ldots, N_{m n}^{(t)}(u)\right\}\right)$, the history generated during $[t, t+u]$ by the claim,

$y_{m n}(u)=\mathrm{E} Y_{m n}^{(t)}(u)$, the average claim amount paid at time $t+u$ if a transition from $m$ to $n$ occurs at that time,

$\sigma_{m n}^{2}(u)=\operatorname{Var} Y_{m n}^{(t)}(u)$, the variance on the claim amount paid at time $t+u$ if a transition from $m$ to $n$ occurs at that time. 
We shall also make use of the fact that

$$
d N_{m n}^{(t)}(u)=I_{m}^{(t)}(u-) \lambda_{m n}(u) d u+d M_{m n}^{(t)}(u),
$$

where $u$ - denotes the left-hand limit, and all $M_{m n}^{(t)}(u)$ for $m, n \in S$ and $m \neq n$ are mutually orthogonal zero-mean martingales with respect to the internal history of the process $\left\{S^{(t)}(u)\right\}_{u \geq 0}$ (see e.g. ANDERSEN et al. (1985)). Because $\left\{S^{(t)}(u)\right\}_{u \geq 0}$ is stochastically independent of the claim amounts according to assumption $(b)$, it is also true that $M_{m n}^{(t)}(u)$ is a zero-mean martingale with respect to the filtration $\left\{\mathcal{H}_{u}^{(t)}\right\}_{u} \geq 0$. Furthermore,

$$
\operatorname{Var}\left[d M_{m n}^{(t)}(u) \mid \mathcal{H}_{u-}^{(t)}\right]=I_{m}^{(t)}(u-) \lambda_{m n}(u) d u .
$$

Let $X^{(t)}(u, v)$ denote the total payment made during $\left.] t+u, t+v\right]$ in respect of a claim incurred at time $t$. We may write $X^{(t)}(u, v)$ as

$$
X^{(t)}(u, v)=\sum_{m \neq n} \int_{u}^{\nu} Y_{m n}^{(t)}(\xi) d N_{m n}^{(t)}(\xi) .
$$

We make the convention that $0 \in S$, and that this state represents IBNR claims. Also $\Delta \in S$, and $\Delta$ is an absorbing state representing fully settled claims. With this convention the number of claims incurred during $[0, t]$, which at time $\tau$ are classified as IBNR and RBNS claims, respectively, can be written as

$$
\begin{gathered}
K_{I B N R}(t)=\int_{0}^{t} I_{0}^{(s)}(\tau-s) d K(s), \\
K_{R B N S}(t)=\int_{0}^{t}\left[1-I_{0}^{(s)}(\tau-s)-I_{\Delta}^{(s)}(\tau-s)\right] d K(s),
\end{gathered}
$$

and the corresponding outstanding (at time $\tau$ ) claims payments are

$$
\begin{aligned}
& X_{I B N R}(t)=\int_{0}^{t} X^{(s)}(\tau-s, \infty) d K_{I B N R}(s), \\
& X_{R B N S}(t)=\int_{0}^{t} X^{(s)}(\tau-s, \infty) d K_{R B N S}(s) .
\end{aligned}
$$

In Section 4 we derive expressions for the IBNR and RBNS reserves, defined as the expected claims payments $X_{I B N R}(\tau)$ and $X_{R B N S}(\tau)$ given the available information at time $\tau$, and the corresponding prediction errors. Before we proceed to do so, we shall in Section 3 derive the required moments of the future payments $X^{(s)}(\tau-s, \infty)$ in respect of a single claim.

\section{FUTURE PAYMENTS ON A SINGLE CLAIM}

Consider the claims payments $X^{(t)}(u, \infty)$ in respect of a single claim incurred at time $t$, as defined in (2.3). We shall derive expressions for the conditional moments 
of $X^{(t)}(u, \infty)$, given the individual history $\mathcal{H}_{u}^{(t)}$ of that claim. Since all quantities considered here are functions of the mark $Z^{(t)}$ corresponding to a claim incurred at time $t$, and the distribution of $Z^{(t)}$ does not depend on $t$ according to assumption (b), we may in this section omit the superscript $(t)$.

Consider the conditional distribution of $X(u, \infty)$ given $\mathcal{H}_{u}$. By the independence assumed in (b), the information about past claim amounts $Y_{m n}(v)$ for $v \leq u$ may be omitted from the history $\mathcal{H}_{u}$. From the Markov property it furthermore follows that the only information contained in $\mathcal{H}_{u}$ about the future development of $\{S(v)\}$ is the present state $S(u)$. Thus,

$$
\begin{aligned}
\mathrm{E}\left[X(u, \infty) \mid \mathcal{H}_{u}\right] & =\mathrm{E}[X(u, \infty) \mid S(u)]:=V(u \mid S(u)), \\
\operatorname{Var}\left[X(u, \infty) \mid \mathcal{H}_{u}\right] & =\operatorname{Var}[X(u, \infty) \mid S(u)]:=\Gamma(u \mid S(u)) .
\end{aligned}
$$

With $X(u, \infty)$ given by (2.3) we obtain by independence of $\{S(u)\}_{u \geq 0}$ and $\left\{Y_{m n}(u)\right\}_{u \geq 0}$, and by use of the decomposition (2.1), that

$$
\begin{aligned}
V(u \mid j) & =\mathrm{E}[X(u, \infty) \mid S(u)=j] \\
& =\sum_{m \neq n} \int_{u}^{\infty} \mathrm{E}\left(Y_{m n}(\xi) \mid S(u)=j\right) \mathrm{E}\left(d N_{m n}(\xi) \mid S(u)=j\right) \\
& =\sum_{m \neq n} \int_{u}^{\infty} y_{m n}(\xi) \mathrm{E}\left[I_{m}(\xi-) \lambda_{m n}(\xi)+d M_{m n}(\xi) \mid S(u)=j\right] \\
& =\sum_{m \neq n} \int_{u}^{\infty} y_{m n}(\xi) p_{j m}(u, \xi) \lambda_{m n}(\xi) d \xi, \quad j \in \mathcal{S},
\end{aligned}
$$

where the latter equality in (3.3) follows by noting that

$$
\mathrm{E}\left[d M_{m n}(\xi) \mid S(u)=j\right]=\mathrm{E}\left\{\mathrm{E}\left[d M_{m n}(\xi) \mid \mathscr{H}_{u}\right] \mid S(u)=j\right\}=0
$$

for $\xi \geq u$, because $\left\{M_{m n}(u)\right\}_{u \geq 0}$ is a martingale with respect to the history of that claim.

For the purpose of deriving formulas for the variance functions $\Gamma(u \mid j)$ in (3.2), we shall find it convenient to work will the loss corresponding to $] u, v]$, defined as

$$
L(u, v)=X(u, v)+V(v \mid S(v))-V(u \mid S(u)) .
$$

The loss as defined in (3.4) plays a key role in connection with results of Hattendorff-type in life-insurance (e.g. PAPATRIANDAFYlOU \& WATERS, 1984) due to the fact that $\{L(u, v)\}_{v \geq u}$ is a zero-mean martingale with respect to $\left\{\mathcal{H}_{v}\right\}_{v \geq u}$. This is most easily seen by writing

$$
L(u, v)=\mathrm{E}\left[X(0, \infty) \mid \mathscr{H}_{\nu}\right]-\mathrm{E}\left[X(0, \infty) \mid \mathscr{H}_{u}\right], v \geq u,
$$

which for $u \leq \xi \leq v$ shows that

$$
\begin{aligned}
\mathrm{E}\left(L(u, v) \mid \mathcal{H}_{\xi}\right) & =\mathrm{E}\left\{\mathrm{E}\left[X(0, \infty) \mid \mathscr{H}_{v}\right] \mid \mathcal{H}_{\xi}\right\}-\mathrm{E}\left[X(0, \infty) \mid \mathscr{H}_{u}\right] \\
& =\mathrm{E}\left[X(0, \infty) \mid \mathscr{H}_{\xi}\right]-\mathrm{E}\left[X(0, \infty) \mid \mathscr{H}_{u}\right]=L(u, \xi) .
\end{aligned}
$$


Because of (3.4), with $v=\infty$, and because the increments of a martingale are uncorrelated, we may then calculate the conditional variance (3.2) as

$$
\begin{aligned}
\operatorname{Var}\left(X(u, \infty) \mid \mathcal{H}_{u}\right) & =\operatorname{Var}\left(L(u, \infty) \mid \mathcal{H}_{u}\right) \\
& =\int_{u}^{\infty} \operatorname{Var}\left(L(d \xi) \mid \mathcal{H}_{u}\right),
\end{aligned}
$$

where $L(d \xi)$ is a short-hand for the loss corresponding to an infinitesimal interval containing $\xi$. An expression for $\operatorname{Var}\left(L(d \xi) \mid \mathcal{H}_{u}\right)$ may be obtained using calculations similar to those in NORBERG (1992). According to (3.4) it holds that

$$
L(d \xi)=\sum_{m \neq n} Y_{m n}(\xi) d N_{m n}(\xi)+d V(\xi \mid S(\xi))
$$

By writing

$$
V(\xi \mid S(\xi))=\sum_{m} I_{m}(\xi) V(\xi \mid m)
$$

we obtain that

$$
d V(\xi \mid S(\xi))=\sum_{m}\left\{V(\xi \mid m) d I_{m}(\xi)+I_{m}(\xi)\left(\frac{d}{d \xi} V(\xi \mid m)\right) d \xi\right\} .
$$

Since $I_{m}(\xi)$ increases by one if a transition into state $m$ is made at time $\xi$ and decreases by one if a transition out of state $m$ is made at that time, we may write

$$
d I_{m}(\xi)=\sum_{n: n \neq m} d N_{n m}(\xi)-\sum_{n: n \neq m} d N_{m n}(\xi) .
$$

The reserve $V(\xi \mid m)$ is a prospective reserve for a Markov model, as used in classical life-insurance mathematics. In the present case, the reserve (3.3) contains no interest or premium payments, and Thiele's differential equation then becomes,

$$
\frac{d}{d \xi} V(\xi \mid m)=-\sum_{n: n \neq m} \lambda_{m n}(\xi) r_{m n}(\xi)
$$

where

$$
r_{m n}(\xi)=y_{m n}(\xi)+V(\xi \mid n)-V(\xi \mid m)
$$

denotes the (expected) sum at risk at time $\xi$. Combining (3.7) with (3.8) and (3.9) yields

$$
\begin{aligned}
d V(\xi \mid S(\xi)) & =\sum_{m \neq n} V(\xi \mid m)\left(d N_{n m}(\xi)-d N_{m n}(\xi)\right)-I_{m}(\xi) \lambda_{m n}(\xi) r_{m n}(\xi) d \xi \\
& =\sum_{m \neq n}(V(\xi \mid n)-V(\xi \mid m)) d N_{m n}(\xi)-I_{m}(\xi) \lambda_{m n}(\xi) r_{m n}(\xi) d \xi
\end{aligned}
$$


Integrating (3.6) from $u$ to $v$ with $d V(\xi \mid S(\xi))$ given above, we then arrive at the expression

$$
\begin{aligned}
L(u, v)= & \sum_{m \neq n} \int_{u}^{v}\left[Y_{m n}(\xi)+V(\xi \mid n)-V(\xi \mid m)\right] d N_{m n}(\xi)- \\
& -\sum_{m \neq n} \int_{u}^{v} I_{m}(\xi) \lambda_{m n}(\xi) r_{m n}(\xi) d \xi
\end{aligned}
$$

Since the latter integral in (3.1) is an ordinary Lebesgue integral, we may here replace $I_{m}(\xi)$ with its left-hand limit $I_{m}(\xi-)$, which allows the alternative expression

$$
L(u, v)=\sum_{m \neq n} \int_{u}^{v} \bar{Y}_{m n}(\xi) d N_{m n}(\xi)+\sum_{m \neq n} \int_{u}^{v} r_{m n}(\xi) d M_{m n}(\xi),
$$

where $M_{m n}(\xi)$ is the martingale (2.1) and

$$
\bar{Y}_{m n}(\xi)=Y_{m n}(\xi)-y_{m n}(\xi) \text {. }
$$

For the purpose of calculating the conditional variance (3.5), the expression (3.12) is useful.

The terms $\bar{Y}_{m n}(\xi) d N_{m n}(\xi)$ are mutually uncorrelated given $\mathcal{H}_{u}$, as a consequence of assumption (b), and

$$
\begin{aligned}
\operatorname{Var}\left[\bar{Y}_{m n}(\xi) d N_{m n}(\xi) \mid \mathcal{H}_{u}\right] & =\mathrm{E}\left[\bar{Y}_{m n}(\xi)^{2} d N_{m n}(\xi)^{2} \mid \mathcal{H}_{u}\right] \\
& =\sigma_{m n}^{2}(\xi) p_{S(u) m}(u, \xi) \lambda_{m n}(\xi) d \xi
\end{aligned}
$$

The terms $r_{m n}(\xi) d M_{m n}(\xi)$ are mutally uncorrelated because the martingales $M_{m n}(\xi)$ are, and by use of (2.2),

$$
\operatorname{Var}\left[r_{m n}(\xi) d M_{m n}(\xi) \mid \mathcal{H}_{u}\right]=r_{m n}(\xi)^{2} p_{S(u) m}(u, \xi) \lambda_{m n}(\xi) d \xi .
$$

Finally, the terms $\bar{Y}_{m n}(\xi) d N_{m n}(\xi)$ and $r_{m n}(\xi) d M_{m n}(\xi)$ are uncorrelated as a consequence of assumption (b). From (3.5), (3.12) and the above expressions we then obtain that the variance functions $\Gamma(u \mid j)$ appearing in (3.2) can be expressed as

$$
\Gamma(u \mid j)=\sum_{m \neq n} \int_{u}^{\infty} p_{j m}(u, \xi) \lambda_{m n}(\xi)\left[\sigma_{m n}^{2}(\xi)+r_{m n}(\xi)^{2}\right] d \xi
$$

In the context of life insurance, variance formulas analogous to (3.13) were obtained by RAMLAU-HANSEN (1988) for a Markov model and by NoRBERG (1992) in a more general counting process framework. However, in life insurance the size of the benefits is specified in the insurance contract, and these are consequently considered as deterministic. The variance $\sigma_{m n}^{2}(\xi)$ does therefore not appear in the formulas of RAMLAU-HANSEN (1988) and NORBERG (1992). 
Remark 3.1. To obtain tables of $V(u \mid j)$ and $\Gamma(u \mid j)$ from (3.3) and (3.13), respectively, one has to calculate first the transition probabilities $p_{j m}(u, v)$ by solving Kolmogorov's differential equations. A computationally more convenient approach is to calculate $V(u \mid j)$ directly by solving Thiele's differential equation (3.9) with boundary conditions $V(\infty \mid j)=0$. In practice one will of course use a boundary condition $V\left(u_{\max } \mid j\right)=0$, where $u_{\max }$ is chosen such that all claims are fully settled within the first $u_{\max }$ time units after occurrence. Comparing (3.3) and (3.13) shows that the formula (3.13) can be obtained from (3.3) by replacing the average claim amount $y_{m n}(\xi)$ by $\sigma_{m n}^{2}(\xi)+r_{m n}(\xi)^{2}$. Taking the derivative with respect to $u$ it then follows that $\Gamma(u \mid j)$ satisfies a Thiele's differential equation (3.9), except that $\sigma_{m n}^{2}(u)+r_{m n}(u)^{2}$ replaces $y_{m n}(u)$ also in this case. Thus,

(3.14) $\frac{d}{d u} \Gamma(u \mid j)=-\sum_{m: m \neq j} \lambda_{j m}(u)\left[\sigma_{j m}^{2}(u)+r_{j m}(u)^{2}+\Gamma(u \mid m)-\Gamma(u \mid j)\right]$,

and $V\left(\left.u\right|_{j}\right)$ as well as $\Gamma(u \mid j)$ may be calculated without necessarily calculating the transition probabilities.

\section{CLAIMS RESERVES}

By time $\tau$ we have registered all known (reported) claim occurrences during $[0, \tau]$, and for a reported claim incurred at time $t$, say, we have also registered the individual history $\mathcal{H}_{\tau-t}^{(t)}$ of that claim from the time of occurrence up to present time $\tau$. Let $\mathcal{F}_{t}$ denote the collection of this information.

The IBNR and RBNS reserves at time $\tau$ are defined as

$$
\begin{aligned}
& V_{I B N R}(\tau)=\mathrm{E}\left(X_{I B N R}(\tau) \mid \mathcal{F}_{\tau}\right), \\
& V_{R B N S}(\tau)=\mathrm{E}\left(X_{R B N S}(\tau) \mid \mathcal{F}_{\tau}\right),
\end{aligned}
$$

where $X_{I B N R}(t)$ and $X_{R B N S}(t)$ are defined in (2.6) and (2.7). The corresponding prediction errors are denoted by

$$
\begin{aligned}
& \Gamma_{I B N R}(\tau)=\operatorname{Var}\left(X_{I B N R}(\tau) \mid \mathcal{F}_{\tau}\right), \\
& \Gamma_{R B N S}(\tau)=\operatorname{Var}\left(X_{R B N S}(\tau) \mid \mathcal{F}_{\tau}\right) .
\end{aligned}
$$

Considering RBNS claims, the occurrences $\left\{K_{R B N S}(t)\right\}_{0 \leq t \leq \tau}$ are known from $\mathcal{F}_{\tau}$, and from (2.7) we then obtain

$$
V_{R B N S}(\tau)=\int_{0}^{\tau} \mathrm{E}\left(X^{(t)}(\tau-t, \infty) \mid \mathcal{F}_{\tau}\right) d K_{R B N S}(t)
$$

By assumption (a) the conditional expectation appearing in (4.5) depends only on the history $\mathcal{H}_{t-t}^{(t)}$ of that particular claim, and from (3.1) we then have,

$$
V_{R B N S}(\tau)=\int_{0}^{\tau} V\left(\tau-t \mid S^{(t)}(\tau-t)\right) d K_{R B N S}(t),
$$


where $V\left(\left.u\right|_{j}\right)$ is the reserve (3.3). By independence of the marks corresponding to different claims we also have,

$$
\begin{aligned}
\Gamma_{R B N S}(\tau) & =\int_{0}^{\tau} \operatorname{Var}\left[X^{(t)}(\tau-t, \infty) \mid \mathcal{F}_{\tau}\right] d K_{R B N S}(t) \\
& =\int_{0}^{\tau} \Gamma\left(\tau-t \mid S^{(t)}(\tau-t)\right) d K_{R B N S}(t),
\end{aligned}
$$

where $\Gamma(u \mid j)$ is defined in (3.13). Note that the integrals in (4.6), (3.13) simply represent summation over those claims which are RBNS at time $\tau$. Thus, the RBNS reserve (4.6) is obtained by adding the reserves $V(u \mid j)$ corresponding to the current states and durations for the RBNS claims at time $\tau$.

From (2.4) and (2.5) we note that $\left\{K_{I B N R}(t)\right\}_{0 \leq t \leq \tau}$ and $\left\{K_{R B N S}(t)\right\}_{0 \leq t \leq \tau}$ are obtained as a marker dependent partition of the Poisson process $\{K(t)\}$. From Norberg (1993, Theorem 2) it then follows that the marked point processes corresponding to $\left\{K_{I B N R}(t)\right\}_{0 \leq t \leq \tau}$ and $\left\{K_{R B N S}(t)\right\}_{0 \leq t \leq \tau}$ are independent and (again) Poisson. The Poisson rate corresponding to IBNR claims is given by

$$
\mu_{I B N R}(t)=\mu(t) \mathrm{P}\left(S^{(t)}(\tau-t)=0\right)=\mu(t) p_{(0)}(\tau-t)
$$

and the mark corresponding to an IBNR claim incurred at time $t$ is distributed according to the conditional distribution of $Z^{(t)}$ given that $S^{(t)}(\tau-t)=0$. Since the history $\mathcal{F}_{\tau}$ is generated by reported claims (only), it then also follows that $X_{I B N R}(t)$ is independent of $\mathcal{F}_{\tau}$, and from (4.1), (2.6) we obtain that

$$
\begin{aligned}
V_{I B N R}(\tau) & =\mathrm{E} X_{I B N R}(\tau) \\
& =\int_{0}^{\tau} \mu_{I B N R}(t) \mathrm{E}\left(X^{(t)}(\tau-t, \infty) \mid S^{(t)}(\tau-t)=0\right) d t \\
& =\int_{0}^{\tau} \mu_{I B N R}(t) V(\tau-t \mid 0) d t,
\end{aligned}
$$

and

$$
\begin{aligned}
\Gamma_{I B N R}(\tau) & =\operatorname{Var} X_{I B N R}(\tau) \\
& =\int_{0}^{\tau} \mu_{I B N R}(t) \mathrm{E}\left(X^{(t)}(\tau-t, \infty)^{2} \mid S^{(t)}(\tau-t)=0\right) d t \\
& =\int_{0}^{\tau} \mu_{I B N R}(t)\left[\Gamma(\tau-t \mid 0)+V(\tau-t \mid 0)^{2}\right] d t .
\end{aligned}
$$

We have now derived formulas for the IBNR and RBNS reserves (4.6), (4.8), and the corresponding prediction errors (4.7), (4.9), expressed in terms of the 
reserve- and variance functions (3.3) and (3.13). The total reserve is (of course) the sum of IBNR and RBNS reserves. Because the marked point processes corresponding to $\left\{K_{I B N R}(t)\right\}$ and $\left\{K_{R B N S}(t)\right\}$ are stochastically independent, it also holds that the prediction error corresponding to the total reserve is obtained by adding the prediction errors corresponding to the IBNR and RBNS components.

Remark 4.1. If $V(u \mid j)$ and $\Gamma(u \mid j)$ are calculated directly by solving Thiele's differential equation as advocated in Remark 3.1, one also needs an expression for $p_{00}(0, u)$ in order to calculate (4.8) and (4.9). However, since state 0 is strongly transient, we have the expression

$$
p_{00}(0, u)=\exp \left\{-\int_{0}^{u} \sum_{m \neq 0} \lambda_{0 m}(\xi) d \xi\right\} .
$$

\section{REFERENCES}

Andersen, P.K. and Borgan, $\emptyset$. (1985) Counting process models for life history data: A review (with discussion). Scan. Journ. Stat, 12, 97-158.

ARJAS, E. (1989) The claims reserving problem in non-life insurance: Some structural ideas. ASTIN Bulletin 19, 139-152.

Hachemeister, C. A. (1980) A stochastic model for loss reserving. Proceedings. ICA 1980, 185-194.

NoRBERG, R. (1992) Hattendorff's theorem and Thiele's differential equation generalized. Scand. Actuarial J., 1992, 2-14.

NorberG, R. (1993) Prediction of outstanding liabilities in non-life insurance. ASTIN Bulletin 23, 95-115.

Papatriandafyloy, A. and Waters, H.R. (1984) Martingales in life insurance. Scand. Actuarial J., $1984,210-230$.

RamlaU-Hamsen, H. (1988) Hattendorff's theorem: A Markov chain and counting process approach. Scand. Actuarial J., 1988, $143-156$.

\section{Ole Hesselager}

Laboratory of Actuarial Mathematics, Universitetsparken 5, University of Copenhagen, DK-2100 Copenhagen $\emptyset$. 